\title{
Effect of time and space uncertainty on a vigilance task*
}

\author{
STANIŠA MILOŠEVIĆ \\ Institute of Psychology, Belgrade, Yugoslavia
}

\begin{abstract}
Two groups, each of $12 \mathrm{Ss}$, were instructed to detect increments in light level from displays of one or five lights. Signals appeared in regular and in irregular intervals. The display was flashed on simultaneously for $0.8 \mathrm{sec}$ every $3.2 \mathrm{sec}$. Signal detection was superior with a display of one light than with a display of five lights. For two extreme response criteria, there was a decrement of false alarms and an increment of beta during a run. These changes were not dependent upon time and spatial uncertainty. There were no systematic changes in correct detections and $d^{\prime}$ during a run. No difference was found between central and peripheral light positions. Correlation of measures was also examined.
\end{abstract}

The theory of signal detectability (Tanner \& Swets, 1954) was initially applied to vigilance situations by Egan, Greenberg, and Schulman (1961). In the present experiment, the effects of spatial and temporal uncertainty are tested within the TSD framework.

According to expectancy theory (Deese, 1955; Baker, 1963), the regularity of signals is an important factor in their detection and strongly affects detection probability during a run. Comparing three programs of signal presentation, Baker found overall performance to be poorest and the decline in detection strongest when the signals were most irregular. Later studies, using a reaction time measure as cited by Davies and Tune (1970), did not support this contention. On the other hand, the effects of spatial uncertainty on overall performance are more significant. Mackworth (1950) found that the effects of spatial uncertainty were stronger than those of temporal uncertainty. Adams and Boulter (1964) came to the same conclusion, using a reaction time task. Ss react slowest when they are uncertain as to where and when signals will occur. They found performance decrements only with temporal uncertainty. Other experiments using spatial uncertainty also have found no changes in simple detection measures as a function of time on task (Broadbent, 1958; Jerison, 1963; Howland \& Wiener, 1963; Broadbent \& Gregory, 1965). However, Wiener (1964), Johnston et al (1966), and Goldstein et al (1969) found some evidence of decrement of performance during a run. After reviewing the literature, Mackworth states, "It can therefore be concluded that while spatial uncertainty has a considerable effect on the overall ability to detect and respond to a signal, its effect on the decrement on performance during the session is less well established than that of temporal uncertainty [1970, p. 104]."

The present experiment used TSD rating scale measures to compare the effects of temporal and spatial

*This paper is a part of the author's PhD dissertation at the Faculty of Philosophy at the University of Belgrade. The author would like to express his appreciation to Martin Taylor and Irwin Pollack for helpful comments and suggestions for this paper. uncertainty in the detection of an increment in the brightness of a flashed light.

\section{METHOD}

\section{Apparatus}

An electronic programmer controlled the display of signals according to one of four programs, having different spatial and temporal characteristics. The display consisted of a square, $25 \mathrm{~cm}$ on a side, covered with black flannel, in which were placed five lights, four at the corners of a concentric square, $18 \mathrm{~cm}$ on a side, and one at the center. The lights were $12-\mathrm{V}$ bulbs behind 9-mm-diam disks of opalescent glass. The bulbs were set into the display. For the nonsignal background level, the voltage across the bulbs was $4.18 \mathrm{~V}$; for the signal level, the voltage was $5.21 \mathrm{~V}$.

\section{Subjects}

Twenty-four students took part in the experiment. Their ages ranged from 17 to 33 years, averaging 20 years. The experiment was part of their trequired studies.

Tasks

The five lights, or the central light only, flashed on rhythmically for $0.8 \mathrm{sec}$ every $3.2 \mathrm{sec}$. The $S$ was required to detect the occurrence of the slightly brighter flash. Signals occurred with 2 deg of spatial uncertainty-on the central light only, or on any one of the five lights-and with $2 \mathrm{deg}$ of temporal uncertainty-every $120 \mathrm{sec}$, or after an interval of 40 , 120 , or $200 \mathrm{sec}$. Four tasks are defined by combining these variables: Task I-center light, every $120 \mathrm{sec}$; Task II-center light, irregularly; Task III-any light, every $120 \mathrm{sec}$; Task IV-any light, irregularly.

Each experimental run lasted $1 \mathrm{~h}$. Ten signals were presented in each 20-min period. In Tasks II and IV, with irregular presentation, the signals appeared at 2, 2:40, 6, 6:40, 8:40, 12, $14,14: 40,18$, and $20 \mathrm{~min}$ into each $20-\mathrm{min}$ period. This timing provides 9 intervals of $40 \mathrm{sec}$ and of $200 \mathrm{sec}$ and 12 intervals of $120 \mathrm{sec}$ during the hour. In Tasks III and IV, during the experimental run, lasting $1 \mathrm{~h}$, six signals were presented at each of the five lights.

Ss rated their responses according to the certainty with which they believed a signal to have just occurred, by pressing one of three buttons on a keyboard. The buttons were labeled "sure it was a signal," "not quite sure," and "can hardly decide, but think it was a signal." Responses within 4 sec after the onset of a signal were considered to be correct detections; the others were considered as false alarms. 


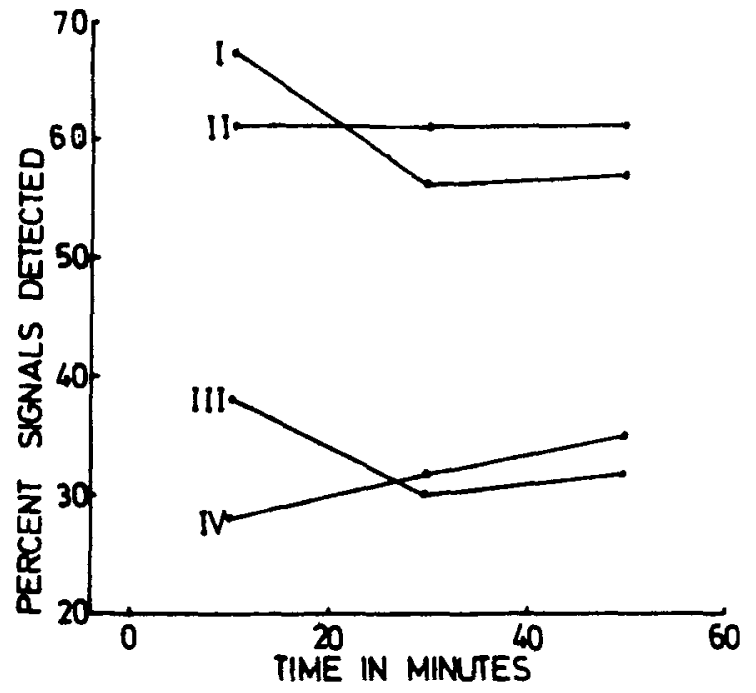

Fig. 1. Percentage of signals detected on a run for strict response criterion in four tasks

\section{Procedure}

Ss were tested individually in an anechoic chamber lit by a single $300-\mathrm{W}$ bulb. Their eyes were $1 \mathrm{~m}$ from, and level with, the center of the display. The keyboard was held in their hands. Each $\mathbf{S}$ was given a short demonstration period lasting usually about $10 \mathrm{~min}$, but sometimes as long as $40 \mathrm{~min}$. During the demonstration, $S$ practiced discriminating signals from background flashes. Later, $S$ was given a 6 -min practice session with the signal rate which corresponded to that in experimental sessions. If $\mathbf{S}$ missed all the signals, the practice session was repeated after further demonstration. Ss were informed after the practice session whether or not they had correctly detected any signals, but were not informed how many signals they had detected or how many false alarms they had made. Since TSD measures were to be employed, it was necessary that $S$ give enough false alarms for later analysis, but not so many as to swamp the hits. Ss were therefore warned to be more cautious or more relaxed if they had given too many or too few false alarms during the practice session.

The experimental design was a 2 by 2 by 2 factorial-Spatial Uncertainty by Temporal by $20-\mathrm{min}$ Period-with repeated measures on the last two factors. Two groups of $12 \mathrm{Ss}$ each define the first factor, spatial uncertainty. One group worked in the single-light tasks and the other in the five-light tasks.

\section{RESULTS}

The results are considered in terms of both the simple detection probabilities, hits and false alarms, and the TSD measures, $\mathrm{d}^{\prime}$ and beta. The analysis considers only the two extreme criteria, strict and lax, although the Ss consistently distinguished all three criteria. Hits and false alarms for the lax criterion were determined by adding those for all three response criteria, under the usual assumption for rating scale procedures. False alarm probabilities were determined from the ratio of the number of false alarms to the number of background flashes. The interpolation technique of Jerison et al (1965) was applied in situations when there were no detected signals, no errors, or when all the signals were detected. Such corrections were rarely needed in this experiment.

\section{Simple Detection Measures}

Correct Detection. Figure 1 shows the percentage of signals correctly detected in the three 20-min periods for the four tasks, considering only the strict criterion. Analysis of variance after arc-sine transformation shows that there are no important differences between criteria over the course of a run. Figure 1 may be considered as representative, although, of course, the lax criterion gave more correct detections. For the strict criterion, analysis of variance shows interactions between regularity and time period $(p<.05)$ and between spatial uncertainty, regularity, and time period $(p<.01)$. For either criterion, the tasks with the single light gave more detections than the tasks with five lights $(p<.01)$.

False Alarms. The percentages of false alarms for the four tasks in the three 20-min periods are shown in Fig. 2, for the lax criterion. The same trend is evident in the data for the strict criterion, but with fewer false alarms. False alarms become less frequent over the course of the run $(p<.01$ for both criteria). There were no other significant effects or interactions, and the decline can be said to be independent of spatial or temporal uncertainty.

\section{Signal Detection Theory Analysis}

Sensitivity, d'. Changes in $d^{\prime}$ over the session are shown for the tasks and a lax criterion in Fig. 3. There is evidently no consistent decline in sensitivity over the run, and analysis of variance shows no significant effects or interactions other than the reduced sensitivity associated with spatial uncertainty $(p<.05$ for the lax criterion, $p<.01$ for the strict criterion).

Criterion, Beta. Figure 4 shows the values of $\log$ beta over the course of the run for the four tasks under the lax criterion. Beta increases, i.e., Ss became more cautious, in three of the tasks, but not in Task IV, which used both spatial and temporal uncertainty. Overall, the

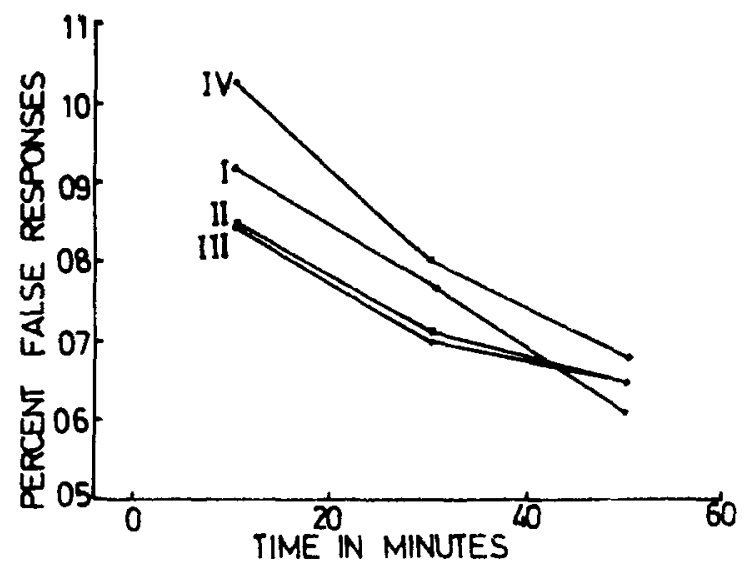

Fig. 2. Percentage of false responses during a nun for the lax response criterion. 
increase in beta is significant $(p<.01)$ for both criteria. The interaction between spatial uncertainty and time period is significant $(p<.05)$ for the lax criterion. Ss apparently are more cautious when they monitor five lights than when they monitor one.

Table 1 shows the average criteria, log beta, used in the four tasks. Although the three response criteria are well distinguished in all tasks, the spread of log beta appears to be greater in the single-light tasks, I and II, than in the five-light tasks, III and IV.

\section{The Effect of Spatial Position on Signal Detection}

In Tasks III and IV, the position of target affected the probability that it would be detected for the medium and strict criteria $\left(\mathrm{p}<.01, \chi^{2}\right.$ test). With the lax criterion, no such positional effects were found. In no case was a difference found between the central and the peripheral signals, and no effect of position on the changes of detection over the run could be demonstrated.

\section{Correlation of Detection in the Tasks with the Single Light and in Tasks with the Five Lights}

Correlations were computed between scores within the single-light tasks, I and II, and within the five-light tasks, III and IV. The rho coefficients for all detection measures and for all response criteria are shown in Table 2. Nearly all the coefficients are positive, thereby suggesting consistent individual differences. A smaller group of correlations is significant. Most of the significant coefficients were obtained on the one-light tasks (Tasks I and II).

From Table 2, three characteristics are noted: First, at the level of the strict criterion, the coefficients of correlation were significant for all detection measures in the single-light tasks but not in the five-light tasks. Second, from the strict to lax criterion, the correlation coefficient for detection measures decreases on single-light tasks and increases on the five-light tasks. Third, at the level of the lax criterion, there are the

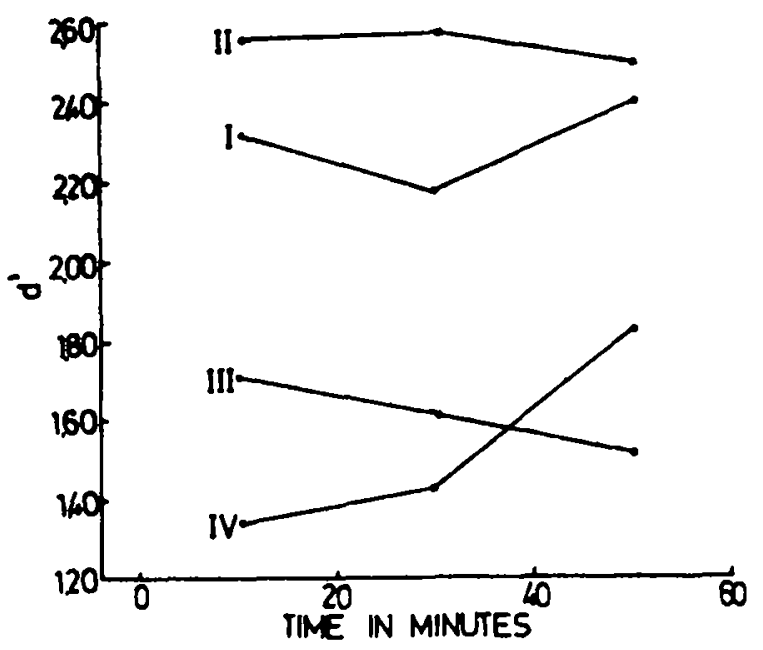

Fig. 3. Index d' during a run for the lax response criterion.

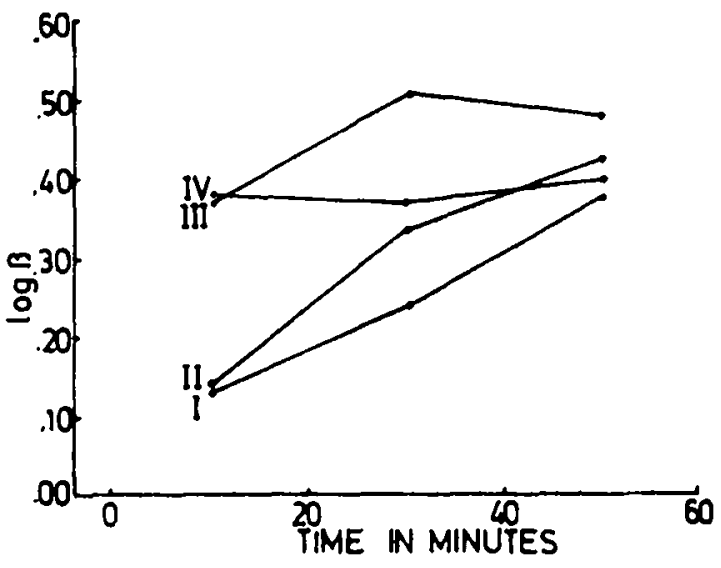

Fig 4. Index $\log$ beta during a run for the lax criterion of response.

Table 1

\begin{tabular}{lrrrr}
$\begin{array}{l}\text { The Average } \begin{array}{l}\log \\
\text { for }\end{array} \\
\text { Three }\end{array}$ in the Vigilance \\
Response & \multicolumn{5}{c}{ Tasks } \\
Criteria & I & II & III & IV \\
\hline Strict & 1.04 & 1.17 & 1.04 & .95 \\
Medium & .48 & .57 & .62 & .60 \\
Lax & .17 & .28 & .48 & .40 \\
\hline
\end{tabular}

differences in the degree of correlation in relation to the detection measures. For this criterion, there are significant correlations in the single-light tasks only between the percentage of correct detections and $d^{\prime}$, while for the five-light tasks only in false alarms and beta.

\section{DISCUSSION}

\section{Temporal vs Spatial Uncertainty}

Under the conditions examined, the sensitivity index, $\mathrm{d}^{\prime}$, does not change consistently during the course of the run, but the response criterion, beta, does increase in three of the four tasks. These data tend to support Broadbent and Gregory (1963) and later workers (e.g., Broadbent \& Gregory, 1965; Jerison et al, 1965; Loeb \& Binford, 1968; Hatfield \& Loeb, 1968; Miloševic, 1969).

The finding of no decrement in $\mathrm{d}^{\prime}$ is in accord with filter theory (Broadbent, 1958). The empty interval in this experiment is rather long, $3.2 \mathrm{sec}$, and Ss might well shift their attention away from the display during this interval without affecting detection. Alternatively, the absence of decrement might be accounted for by the habituation theory, as discussed by Mackworth (1968). With presentations slower than $1 / \mathrm{sec}$, habituation effects might not be manifest. This might be especially true in a visual task in which the $S$ can look around between events, thus reducing habituation effects. The long flash interval, $0.8 \mathrm{sec}$, also may have permitted several eye movements, thereby negating attentional effects.

With respect to the relative contribution of spatial and temporal uncertainty, these results support Mackworth 
Table 2

Rho Coefficients of Correlation of Signal Detection in the Tasks With One or More Signal Sources

\begin{tabular}{|c|c|c|c|c|c|c|c|c|}
\hline \multirow[b]{3}{*}{$\begin{array}{c}\text { Response } \\
\text { Criteria }\end{array}$} & \multicolumn{8}{|c|}{ Measures } \\
\hline & \multicolumn{2}{|c|}{ Correct } & \multicolumn{2}{|c|}{ False Alarms } & \multicolumn{2}{|c|}{$d^{\prime}$} & \multicolumn{2}{|c|}{$\log \beta$} \\
\hline & $\begin{array}{c}\text { I } \\
\text { and } \\
\text { II }\end{array}$ & $\begin{array}{l}\text { III } \\
\text { and } \\
\text { IV }\end{array}$ & $\begin{array}{c}\text { I } \\
\text { and } \\
\text { II }\end{array}$ & $\begin{array}{l}\text { III } \\
\text { and } \\
\text { IV } \\
\end{array}$ & $\begin{array}{c}\text { I } \\
\text { and } \\
\text { II }\end{array}$ & $\begin{array}{l}\text { III } \\
\text { and } \\
\text { IV }\end{array}$ & $\begin{array}{c}\text { I } \\
\text { and } \\
\text { II }\end{array}$ & $\begin{array}{l}\text { III } \\
\text { and } \\
\text { IV }\end{array}$ \\
\hline $\begin{array}{l}\text { Strict } \\
\text { Medium } \\
\text { Lax }\end{array}$ & $\begin{array}{l}.68^{*} \\
.60^{*} \\
.58^{*} \\
\end{array}$ & $\begin{array}{l}.07 \\
.04 \\
.36 \\
\end{array}$ & $\begin{array}{l}.74 \dagger \\
.47 \\
.48\end{array}$ & $\begin{array}{l}.16 \\
.25 \\
.78 \dagger\end{array}$ & $\begin{array}{l}.71 \dagger \\
.66^{*} \\
.60^{*}\end{array}$ & $\begin{array}{r}.05 \\
-.12 \\
.34\end{array}$ & $\begin{array}{l}.75 \dagger \\
.13 \\
.23\end{array}$ & $\begin{array}{l}.23 \\
.28 \\
.75 \dagger\end{array}$ \\
\hline
\end{tabular}

*Significant at the .05 level.

tSignificant at the .01 level.

(1950) and Adams and Boulter (1964) in showing spatial uncertainty to have a stronger effect on overall performance. The relative effects on the course of detection during the run are also in accord with the findings of Adams and Boulter.

The results show no relation between the regularity or irregularity of signal presentation and the time course or overall level of the detection measures, in contradiction to Baker's (1963) version of expectancy theory. On the other hand, in accordance with expectancy theory, spatial uncertainty affects both the overall performance level and the changes in some measures over the course of the run. Detectability is better with a single light than with five. Both lax and strict criteria show differences in beta as a function of spatial uncertainty, and the greater the increase in log beta during a run, the less the spatial uncertainty.

\section{Signal Detection and Spatial Location}

No difference was found between central and peripheral light positions. This accords only with Hockey's (1970) findings and disagrees with Baker (1958), Colquhoun (1961, 1966), and Adams and Boulter (1964). The visual angle between bulbs at opposite corners of the square was $14 \mathrm{deg} 35 \mathrm{~min}$; from the center to the corners, it was $7 \mathrm{deg} 25 \mathrm{~min}$. Since Ss could see the peripheral signals while looking at the center, they might have maintained fixation on the center and monitored all signals from this position. The relatively long signal duration also may have reduced differences between central and peripheral light positions.

\section{Correlation of Performance Measures}

The present study obtained significant correlations in both detection and bias measures, especially for the one-light task at a strict response criterion and for the five-light task at the lax response criterion. Gunn and Loeb (1967) obtained significant cross-modality correlations in both $\mathrm{d}^{\prime}$ and beta. Thus, across tasks, Os show consistent differences in both sensitivity (a cognitive-perceptual component?) and in bias (an attitudinal component?).

\section{REFERENCES}

Adams, J. A., \& Boulter, L. R. Spatial and temporal uncertainty as determinants of vigilance behavior. Journal of Experimental Psychology, 1964, 67, 127-131.

Baker, C. H. Attention to visual displays during a vigilance task. I. Biasing attention. British Journal of Psychology, 1958, 49, 279-288.

Baker, C. H. Farther toward a theory of vigilance. In D. N. Buckner and J. J. McGrath (Eds.), Vigilance: A symposium. New York: McGraw-Hill, 1963. Pp. 127-154.

Broadbent, D. E. Perception and communication. New York: Pergamon Press, 1958.

Broadbent, D. E., \& Gregory, M. Vigilance considered as a statistical decision. British Journal of Psychology, 1963, 54, 309-325.

Broadbent, D. E., \& Gregory, M. Effects of noise and of signal rate upon vigilance analyzed by means of decision theory. Human Factors, 1965, 7, 1955-1962.

Colquhoun, W. P. The effect of "unwanted" signals on performance in a vigilance task. Ergonomics, 1961, 4, 41-52.

Colquhoun, W. P. The effect of "unwanted" signals on performance in a vigilance task: A reply to Jerison. Ergonomics, $1966,9,417-419$.

Davies, D. R., \& Tune, G. S. Human vigilance performance. Lond on: Staple Press, 1970 .

Deese, J. Some problems in the theory of vigilance. Psychological Review, 1955, 62, 359-368.

Egan, J. P., Greenberg. G. L., \& Schulman, A. 1. Operating characteristics. signal detectability and the method of free response. Journal of the Acoustical Society of America, 1961. 33, 993-1007.

Goldstein, I. L., Johnston, W. A., \& Howell, W. C. Complex vigilance: Relevant and irrelevant signals. Journal of Applied Psychology, 1969, 53, 257-268.

Gunn, W. J., \& Loeb, M. Correlation of performance in detecting visual and auditory signals. American Journal of Psychology, $1967,80,236-242$.

Hatfield, J., \& Loeb, M. Sense mode and coupling in a vigilance task. Perception \& Psychophy sics, 1968, 4, 29-36.

Hockey, G. R. J. Signal probability and spatial location as possible base for increased selectivity in noise. Quarterly Journal of Ex perimental Psy chology, 1970, 22, 37-42.

Howland, D., \& Wiener, E. L. The system monitor. In D. N. Buckner and J. J. MeGrath (Eds.). Vigilance: $A$ symposium. New York: McGraw-Hill, 1963. Pp. 217-226.

Jerison, H. J. On the decrement function in human vigilance. In D. N. Buckner and J. J. McGrath (Eds.), Vigilance: $A$ symposium. New York: McGraw-Hill, 1963. Pp. 199-216.

Jerison, H. J., Pickett, R. M., \& Stenson, H. H. The elicited observing rate and decision process in vigilance. Human Factors, 1965, 7, 107-128.

Johnston, W. A., Howell, W. C., \& Goldstein, I. L. Human vigilance as a function of signal frequency and stimulus density. Joumal of Experimental Psychology, 1966, 72, 736-743.

Loeb, M., \& Binford, J. R. Variation in performance on auditory and visual monitoring tasks as a function on signal and stimulus frequencies. Perception \& Psychophysics, 1968, 4, 361-367.

Mackworth, N. H. Research on the measurement of human performance. London: His Majesty's Stationary Office, 1950.

Mackworth, J. F. Vigilance, arousal, and habituation. Psychological R eview, 1968, 75, 308-322.

Mackworth, J. F. Vigilance and attention. A signal detection approach. Harmondsworth: Penguin Books, 1870.

Milosevic, $S$. Détection du signal en function du critère de response. Travail Humain, 1869, 32, 81-86.

Tanner, W. P., \& Swets, J. A. A decision-making theory of visual detection. Psychological Review, 1954,61, 401-409.

Wiener, E. L. Multiple channel monitoring. Ergonomics, 1964, 7, 453-460.

(Received for publication August 13, 1973 ;

final revision recejved November $6,1973$. ) 